\title{
Video Article \\ A Rapidly Incremented Tethered-Swimming Maximal Protocol for Cardiorespiratory Assessment of Swimmers
}

\author{
Dalton M. Pessôa Filho ${ }^{1,2}$, Danilo A. Massini ${ }^{\star^{2}}$, Leandro O. C. Siqueira ${ }^{{ }^{2}}$, Luiz Gustavo A. Santos*², Camila M. T. Vasconcelos ${ }^{*^{2}}$, Tiago A. \\ F. Almeida ${ }^{\star 1,3}$, Mário A. C. Espada ${ }^{\star 4,5}$, Joana F. Reis ${ }^{\star^{3,6}}$, Francisco B. Alves ${ }^{\star^{3}}$, Fred J. DiMenna ${ }^{\star 7,8}$ \\ ${ }^{1}$ Department of Physical Education, São Paulo State University (UNESP) at Bauru \\ ${ }^{2}$ Institute of Bioscience, Graduate Program in Human Development and Technology, São Paulo State University (UNESP) at Rio Claro \\ ${ }^{3}$ Ciper, Faculdade de Motricidade Humana, Universidade de Lisboa \\ ${ }^{4}$ Department of Science and Technology, School of Education, Polytechnic Institute of Setúbal \\ ${ }^{5}$ Quality of Life Research Center, Polytechnic Institute of Santarem \\ ${ }^{6}$ Universidade Europeia at Lisbon \\ ${ }^{7}$ Division of Endocrinology, Diabetes and Bone Disease, Icahn School of Medicine at Mount Sinai \\ ${ }^{8}$ Department of Biobehavioral Sciences, Teachers College, Columbia University \\ *These authors contributed equally
}

Correspondence to: Fred J. DiMenna at dimenna@exchange.tc.columbia.edu

URL: https://www.jove.com/video/60630

DOI: doi:10.3791/60630

Keywords: Biochemistry, Issue 155, incremental exercise testing, cardiorespiratory assessment, swimmers, tethered swimming, maximal rate of oxygen consumption, gas exchange threshold, respiratory compensation point, exercise economy

Date Published: 1/28/2020

Citation: Pessôa Filho, D.M., Massini, D.A., Siqueira, L.O.C., Santos, L.G.A., Vasconcelos, C.M.T., Almeida, T.A.F., Espada, M.A.C., Reis, J.F., Alves, F.B., DiMenna, F.J. A Rapidly Incremented Tethered-Swimming Maximal Protocol for Cardiorespiratory Assessment of Swimmers. J. Vis. Exp. (155), e60630, doi:10.3791/60630 (2020).

\section{Abstract}

Incremental exercise testing is the standard means of assessing cardiorespiratory capacity of endurance athletes. While the maximal rate of oxygen consumption is typically used as the criterion measurement in this regard, two metabolic breakpoints that reflect changes in the dynamics of lactate production/consumption as the work rate is increased are perhaps more relevant for endurance athletes from a functional standpoint.

Exercise economy, which represents the rate of oxygen consumption relative to performance of submaximal work, is also an important parameter to measure for endurance-athlete assessment. Ramp incremental tests comprising a gradual but rapid increase in work rate until the limit of exercise tolerance is reached are useful for determining these parameters. This type of test is typically performed on a cycle ergometer or treadmill because there is a need for precision with respect to work-rate incrementation. However, athletes should be tested while performing the mode of exercise required for their sport. Consequently, swimmers are typically assessed during free-swimming incremental tests where such precision is difficult to achieve. We have recently suggested that stationary swimming against a load that is progressively increased (incremental tethered swimming) can serve as a "swim ergometer" by allowing sufficient precision to accommodate a gradual but rapid loading pattern that reveals the aforementioned metabolic breakpoints and exercise economy. However, the degree to which the peak rate of oxygen consumption achieved during such a protocol approximates the maximal rate that is measured during free swimming remains to be determined. In the present article, we explain how this rapidly incremented tethered-swimming protocol can be employed to assess the cardiorespiratory capacity of a swimmer. Specifically, we explain how assessment of a short-distance competitive swimmer using this protocol revealed that his rate of oxygen uptake was 30.3 and $34.8 \mathrm{~mL} \cdot \mathrm{min}^{-1} \cdot \mathrm{kg}^{-1} \mathrm{BM}$ at his gas-exchange threshold and respiratory compensation point, respectively.

\section{Video Link}

The video component of this article can be found at https://www.jove.com/video/60630/

\section{Introduction}

An exercise test that involves an incremental increase in work rate (WR) from low to maximal (i.e., incremental exercise test; INC) provides the gold standard method of cardiorespiratory assessment for endurance athletes. In addition to the highest WR that the athlete can achieve $\left(W_{\text {peak }}\right)$, INC also allows for determination of the highest rate at which the individual can consume oxygen $\left(\mathrm{O}_{2}\right)$ for that form of exercise $\left({ }^{\mathrm{V}} \mathrm{O}_{2 \text { peak }}\right)$ if gas exchange and ventilatory data are collected during the test ${ }^{1}$. The $\mathrm{V}_{2 \text { peak }}$ represents the criterion measure of cardiorespiratory fitness. Moreover, analysis of gas exchange and ventilatory data collected as WR is increased provides a non-invasive way to identify the point at which blood-lactate concentration (blood [lactate]) increases above the baseline value (lactate threshold) and the point at which it begins to accumulate at an accelerated rate (lactate turnpoint) ${ }^{2}$. These metabolic breakpoints are estimated by determining the gas-exchange threshold (GET) and respiratory-compensation point (RCP), respectively ${ }^{3}$. Importantly, the GET provides a robust estimate of the point at which blood [lactate] initially increases whereas the "hyperventilation" that characterizes RCP is a more complex phenomenon that can be initiated by afferent input other than chemoreception per se. Consequently, conclusions based on identification of RCP should be made with caution. 
When exercise is maintained at a constant rate of work (CWR), there are markedly different physiological response profiles based on the "exercise-intensity domain" within which the WR falls ${ }^{4,5}$. Specifically, achievement of a $\mathrm{VO}_{2}$ and blood [lactate] "steady state" is rapid in the moderate domain, delayed in the heavy domain and unattainable in the severe domain ${ }^{4,5}$. It is well established that the rate at which $\mathrm{O}_{2}$ can be consumed at GET during INC $\left(\mathrm{VO}_{2 \mathrm{GET}}\right)$ serves as the metabolic rate that separates the moderate from heavy domain during CWR $\mathrm{CW}^{3,6}$. Although controversial, a number of recent observations indicate similar equivalence between the rate at which $\mathrm{O}_{2}$ can be consumed at $\mathrm{RCP}\left(\mathrm{V}_{2 \mathrm{RCP}}\right)$ and heavy/severe separation, ${ }^{7,8,9,10}$. Identification of $\dot{\mathrm{V}} \mathrm{O}_{2 \mathrm{GET}}$ and $\mathrm{VO}_{2 \mathrm{RCP}}$ from data collected during INC might, therefore, be useful for prescribing domain-specific training regimens for endurance athletes via metabolic rate with the caveat that aligning a metabolic rate with a specific work rate is more complex than simply doing so according to the $\dot{\mathrm{VO}}_{2}$-work rate relationship derived from the incremental test ${ }^{8,11}$.

When the concept of testing to determine $\mathrm{VO}_{2 \max }$ was initially explored, researchers had subjects perform bouts of track running to the limit of exercise tolerance $\left(\mathrm{T}_{\mathrm{lim}}\right)$ at increasing speeds on separate days ${ }^{1}$. Research followed which confirmed that $\dot{\mathrm{V}}_{2 \text { max }}$ can also be determined from similar bouts performed to $\mathrm{T}_{\lim }$ on the same day with rest periods interspersed ${ }^{12}$. Eventually, it was shown that a continuous protocol with WR increased in an incremental manner at specific time intervals (e.g., every $3 \mathrm{~min}$ ) revealed the same $\mathrm{V}_{2 \text { peak }}$ as the discontinuous tests ${ }^{13}$. Consequently, these "graded exercise tests" became the standard for determining this criterion measure of cardiorespiratory fitness. However, in 1981, Whipp and colleagues published research that indicated that for the purpose of $\mathrm{V}_{2 \max }$ measurement, INC could also be performed entirely in the non-steady state; that is, with WR increasing continuously as a "smooth function of time" (RAMP-INC) ${ }^{14}$. Unlike INC with extended stages and relatively large WR increases per stage, the gradual increase during RAMP-INC ensures that the "isocapnic buffering region" that separates GET and RCP will be clearly defined ${ }^{15}$. Furthermore, much like INC with stages, RAMP-INC can be used to assess "exercise economy" (i.e., the $\mathrm{V}_{2}$ required per given WR); however, unlike INC with stages, in this case, it is the inverse of "delta efficiency" (i.e., the slope of the $\dot{\mathrm{V}}_{2}$-WR relationship) that is used for this purpose ${ }^{11}$ with consideration given to the fact that due to the complexities of the $\dot{V O}_{2}$ response to work rates across the intensity spectrum, this parameter will not be an immutable feature of INC per se (e.g., RAMP-INC initiated from different baseline work rates or characterized by different ramp slopes) or CWR exercise ${ }^{16}$

For general fitness testing, INC is usually performed on a leg ergometer or treadmill because these modalities are more available and leg cycling and walking/running are familiar to the average person. Moreover, administration of RAMP-INC requires the ability to increase WR continuously in small increments (e.g., $1 \mathrm{~W}$ every $2 \mathrm{~s}$ ); hence, an ergometer (typically leg cycling) is best suited for this type of testing. However, athlete assessment is more complex because athletes must be tested while performing the specific mode of exercise required for their sport. For cyclists and individuals who participate in sports that involve running, this is not problematic because of the accessibility and applicability of the aforementioned testing machines. Conversely, ecologically-valid testing with gas exchange and ventilatory data collection and the gradual WR incrementation required for RAMP-INC is more challenging when assessing aquatic athletes.

Prior to the advent of automated collection systems, gas-exchange assessment of swimmers was often performed using Douglas-bag collection following a maximal swim ${ }^{17}$. Once automated systems were developed, "real-time" collection took place, but not under "real-swimming" conditions (e.g., while swimmers swam in a flume which controlled WR) ${ }^{17}$. Unfortunately, the former method has inherent limitations due to the assumptions of "backward extrapolation" while the latter raises concerns regarding the degree to which flume swimming changes technique ${ }^{17}$. The current state of the art involves the use of portable breath-by-breath collection systems which move with the swimmer alongside the pool during free swimming ${ }^{17}$. While this type of measurement improves ecological validity, gradual WR incrementation is challenging. Indeed, INC during free swimming typically involves intervals of set distance (e.g., $200 \mathrm{~m}$ ) at progressively-increasing velocities ${ }^{14,15}$. This means that a test consists of lengthy stages with large unequal WR increments. It is, therefore, not surprising that only a single metabolic breakpoint (typically called the "anaerobic threshold") is reported by researchers who employ this test ${ }^{18,19}$. Instead, we have recently shown that both $\mathrm{VO}_{2 \mathrm{GET}}$ and $\dot{\mathrm{V}} \mathrm{O}_{2 \mathrm{RCP}}$ can be determined from data collected while swimmers performed stationary swimming in a pool against a load that was increased gradually and rapidly (i.e., incremental tethered swimming) ${ }^{20}$. While the unique breathing pattern that is present during swimming might render the aforementioned breakpoints harder to identify compared to typical modes of assessment (personal observation), we believe that this method of testing might be suitable as a "swim ergometer" that can be used for cardiorespiratory assessment of swimmers in a manner similar to how a stationary cycle is used for cyclists. Indeed, we have shown that $\dot{\mathrm{VO}}_{2 \mathrm{GET}}, \dot{\mathrm{VO}}_{2 \mathrm{RCP}}$ and exercise economy (as indicated by the $\dot{\mathrm{V}} \mathrm{O}_{2}$-load slope) can all be determined from the rapidly incremented tethered-swimming protocol that is described below ${ }^{20}$.

\section{Protocol}

Participants in the study from which the representative-subject data presented below were extracted ${ }^{20}(n=11)$ were required to give their written informed consent prior to initiation of testing after the experimental procedures, associated risks and potential benefits of participation had been explained. The first visit comprised a familiarization session during which the swimmers were introduced to the concept of tethered swimming and the measurement techniques that would be in effect during the actual testing. An all-out tethered-swimming test was performed during the second visit and the rapidly incremented tethered-swimming protocol was performed on the third visit. Both tests were done in a semi-Olympic pool $(25 \mathrm{~m})$ with water temperature at $28^{\circ} \mathrm{C}$.

\section{Preparation of swimmer}

1. Instruct the swimmer to avoid strenuous exercise for $24 \mathrm{~h}$ preceding each testing session.

2. Instruct the swimmer to arrive at the pool in a rested and fully hydrated state $\geq 3 \mathrm{~h}$ postprandial.

3. Instruct the swimmer to refrain from ingesting stimulant beverages and alcohol for $24 \mathrm{~h}$ prior to each test.

\section{All-out tethered-swimming test}

1. Prepare the $500 \mathrm{~kg}$ load cell that will be used to measure the highest force that the swimmer can exert during two trials comprising $30 \mathrm{~s}$ of allout swimming ${ }^{21}$.

1. Open the N2000PRO Software (Power Din Pro - CEFISE) program on the computer.

2. Open the Help Menu to verify the communication link between the computer and the load-cell analyzer. 
1. Observe a green signal that indicates that the connection to the RS232 interface is well established.

2. Set the countdown to start the test depending on circumstances.

3. Set the sampling duration. Set the rest interval. Set the frames per second at $100 \mathrm{~Hz}$.

4. Set the unit of force measurement at $\mathrm{N}$ or kg depending on personal preference. Set the acquisition time in milliseconds.

3. Calibrate the load cell ${ }^{22}$ with 0 and $10 \mathrm{~kg}$ loads with the swimmer outside pool.

4. Attach a load cell to the start block via the L-shaped flattened iron bar that is designed by CEFISE specifically for tethered-swimming measurements.

5. Attach one end of the inelastic rope to the load cell and the other end to the swimmer by means of the custom-designed belt (CEFISE), which has ropes attached to both hips such that leg kicking will not interfere with the force measurement.

2. Prepare the swimmer for performance of the two-trial test.

1. Provide instructions to the swimmer regarding correct performance of all-out front-crawl swimming (e.g., prevent the head and trunk from rising while swimming as rapidly as possible, concentrate on kicking at a maximal rate in addition to maximal stroking, etc.).

2. Instruct the swimmer to perform stretching and arm/leg swings at poolside in preparation.

3. Instruct the swimmer to enter the pool and perform a standard warm-up protocol comprised of front-crawl swimming for $800 \mathrm{~m}$ at a light intensity with care taken to avoid engendering any lingering effects that could influence the results of the test.

4. Allow the swimmer to exit the pool and rest at poolside for $10 \mathrm{~min}$.

5. Secure the belt around swimmer's waist. Attach the free end of the inelastic rope to the belt.

6. Determine the load required to maintain the swimmer's body horizontally with a minimum amount of tension on the measurement system (load base).

7. Signal the swimmer to begin Trial \#1 of the test.

3. Monitor the swimmer during performance of the test.

1. Provide verbal encouragement to swimmer throughout $30 \mathrm{~s}$ test.

2. Signal the swimmer to end the test. Detach the swimmer from the inelastic rope.

3. Instruct the swimmer to perform a standard cool-down protocol comprised of front-crawl swimming at a light intensity.

4. Allow the swimmer to rest for $30 \mathrm{~min}$ at poolside.

5. Reattach the swimmer to the inelastic rope.

6. Signal the swimmer to start Trial \#2 of the test which is identical to Trial \#1 ( $30 \mathrm{~s}$ of all-out swimming).

7. Signal the swimmer to end the test.

8. Instruct the swimmer to perform a standard cool-down protocol comprised of front-crawl swimming at a light intensity.

9. Allow the swimmer to exit pool.

4. Analyze the data collected during the two-trial test.

1. Apply the smoothing process to the data using the N2000PRO software package ${ }^{23}$.

2. Calculate the peaks of the wave-frequency signal from the force-time sinusoidal curve (range, sine $\left.80^{\circ}-100^{\circ}\right)$ above load base for Trials \#1 and 2.

3. Define the averaged peaks of the force-time wave-frequency signal in the first $5 \mathrm{~s}$ and entire $30 \mathrm{~s}$, respectively, as the peak force $\left(F_{\text {peak }}\right)$ and average force $\left(F_{\text {avg }}\right)$ for each trial.

4. Use the higher values for $F_{\text {peak }}$ and $F_{\text {avg }}$ for further calculations.

\section{Incremental tethered-swimming test}

1. Calculate the loads that will be used to resist the swimmer's forward displacement during the incremental test.

1. Calculate the starting load as $30 \%$ of the $F_{\text {avg }}$ above load $_{\text {base. }}$.

2. Calculate the increments to be applied per 60 -s stage as $5 \%$ of $F_{\text {avg }}$ above load lase $_{\text {b }}$

2. Prepare the automated portable metabolic unit for data collection.

1. Open the unit's software.

2. Verify the communication link between the computer and the automated portable metabolic unit.

3. Power on unit and allow to warm up for $45 \mathrm{~min}$. Ensure that batteries are fully charged.

4. Perform calibration of unit for environmental air $^{24}$.

5. Perform calibration of unit for reference $\mathrm{O}_{2}(16 \%), \mathrm{CO}_{2}(5 \%)$ and $\mathrm{N}$ (balance) concentrations ${ }^{24}$.

6. Perform mask time-delay calibration ${ }^{24}$.

7. Perform calibration of turbine with $3 \mathrm{~L}$ syringe ${ }^{24}$.

8. Enter the subject data, ambient temperature and humidity.

3. Prepare the swimmer for performance of the incremental test.

1. Install a facemask and a snorkel on the swimmer.

2. Instruct the swimmer to rest at poolside for $10 \mathrm{~min}$ to collect "baseline" gas exchange and ventilatory data.

3. Instruct the swimmer to enter the pool and perform a standard warm-up protocol comprised of front-crawl swimming at a light intensity.

4. Secure a belt around the swimmer's waist. Attach an inelastic rope to the belt with the other end of rope attached to the loading system.

5. Instruct the swimmer that once the test begins to use the two markers on the bottom of the pool for reference points, which allow them to maintain a relatively-fixed position (e.g., $\pm 1 \mathrm{~m}$ from the desired position).

6. Signal the swimmer to begin the test.

4. Monitor the swimmer during performance of the incremental test. 
NOTE: A research assistant who is experienced in monitoring this type of testing should hold the gas-analysis unit at poolside being

cognizant to do so without impeding swimmer displacement and/or elevating the swimmer's head.

1. Increase load while timing the $60 \mathrm{~s}$ stages.

2. Terminate the test and record the time to limit of exercise tolerance when the swimmer is no longer able to maintain the requisite position despite strong verbal encouragement from the testers.

3. Use the time to limit of exercise tolerance to calculate stages completed.

4. Record loads for each stage and peak load.

5. Detach the swimmer from the inelastic rope.

6. Instruct the swimmer to perform a standard cool-down protocol comprised of front-crawl swimming at a low-to-moderate intensity.

7. Allow the swimmer to exit the pool.

5. Analyze the data collected during the incremental test.

1. Smooth breath-by-breath gas-exchange data that were collected before and during test using the unit's software program.

2. Export gas-exchange data in consecutive $9 \mathrm{~s}$ bin averages.

3. Perform three-point rolling average on consecutive $9 \mathrm{~s}$ bin averages for $\mathrm{V}_{2}$.

4. Record highest three-point rolling-average value as the $\dot{\mathrm{V}}_{2 \text { peak }}$.

5. Using final three-point rolling-average value for each completed stage, calculate $\mathrm{V}_{2}$-load relationship via linear regression. Exclude data from end stages of test if a $\mathrm{VO}_{2}$ plateau appears to be present (visual inspection).

6. Using consecutive $9 \mathrm{~s}$ bin averages, determine $\mathrm{VO}_{2 \mathrm{GET}}$.

1. Determine the first disproportionate increase in the rate of $\mathrm{CO}_{2}$ production $\left(\dot{\mathrm{V}} \mathrm{CO}_{2}\right)$ compared to $\mathrm{VO}_{2}$.

2. Determine the increase in the ratio of the expired rate of ventilation $\left(\dot{V}_{E}\right)$ to $\dot{V}_{2}$ with no increase in the ratio of $\dot{V}_{E}$ to $\dot{V} C O_{2}$.

3. Determine the increase in end-tidal $\mathrm{O}_{2}$ tension with no fall in end-tidal $\mathrm{CO}_{2}$ tension.

7. Using consecutive 9-s bin averages, determine $\dot{\mathrm{VO}}_{2 \mathrm{RCP}}$.

1. Determine the first disproportionate increase in $\dot{\mathrm{V}}_{\mathrm{E}}$ compared to $\dot{\mathrm{V}} \mathrm{CO}_{2}$.

2. Determine the decrease in end-tidal $\mathrm{CO}_{2}$.

8. Express $\dot{\mathrm{V}} \mathrm{O}_{2 \text { peak }}, \dot{\mathrm{V}} \mathrm{O}_{2 \mathrm{GET}}, \dot{\mathrm{V}} \mathrm{O}_{2 \mathrm{RCP}}$ and $\dot{\mathrm{V}} \mathrm{O}_{2}$-load slope in both absolute $\left(\mathrm{L} \cdot \mathrm{min}^{-1}\right)$ and relative (to body $\left.\mathrm{mass} \mathrm{mL}^{\mathrm{min}} \mathrm{mi}^{-1} \cdot \mathrm{kg}^{-1}\right)$ terms.

9. Express $\dot{\mathrm{VO}}_{2 \mathrm{GET}}$ and $\mathrm{VO}_{2 \mathrm{RCP}}$ in relative terms as a percentage of $\dot{\mathrm{VO}}_{2 \text { peak. }}$.

\section{Representative Results}

The data presented in Table 1 and depicted in Figures 1-4 represents the response profiles observed for a male swimmer (age, 24 years). At the time of data collection, the swimmer had been training for competitive swimming for 7 years. His specialty was short-distance (i.e., $50 \mathrm{~m}$ and $100 \mathrm{~m}$ ) freestyle events.

The initial load on INC was set at a load that exceeded that which was required for this swimmer to maintain body alignment prior to initiation of the all-out swim $\left(F_{\text {base }}\right)$ by $30 \%$ of the difference between the average force measured during the all-out swim and $F_{\text {base }}(\Delta F)$. For this swimmer, that load was $4.17 \mathrm{~kg}$. The load was then increased by $0.7 \mathrm{~kg}$ for every $60 \mathrm{~s}$ stage (Figure 1). The limit of exercise tolerance for this swimmer occurred at $576 \mathrm{~s}$ (stage 10).

When breath-by-breath $\mathrm{VO}_{2}$ data collected during the baseline and exercise portions of INC were averaged into consecutive $9 \mathrm{~s}$ bins, the highest three-point rolling average was $3.44 \mathrm{~L} \cdot \mathrm{min}^{-1}\left(40.6 \mathrm{~mL} \cdot \mathrm{min}^{-1} \cdot \mathrm{kg}^{-1} \mathrm{BM}\right)\left(\right.$ Figure 2) and the $\dot{\mathrm{V}} \mathrm{O}_{2}$-load slope was $261 \mathrm{~mL} \cdot \mathrm{min}^{-1} \cdot \mathrm{kg}^{-1}(3.1$ $\mathrm{mL} \cdot \mathrm{min}^{-1} \cdot \mathrm{kg}^{-1} \mathrm{BM} \cdot \mathrm{kg}^{-1}$ ) (Figure 3). Identification of GET and RCP were made by consensus from a panel of independent reviewers experienced at making these determinations from a cluster of measurements. Being that GET represents the point during INC at which ventilation changes due to metabolic acidosis and the increased $\dot{\mathrm{CCO}}_{2}$ that occurs consequent to its buffering (i.e., passage of the "lactate threshold"), it can be identified by an increase in the ratio of both $\dot{\mathrm{VCO}}_{2}$ and $\dot{\mathrm{V}}_{\mathrm{E}}$ to $\dot{\mathrm{VO}}_{2}$ that causes end-tidal $\mathrm{O}_{2}$ to increase. However, for data collected during INC with sufficient sensitivity, this change in gas-exchange and ventilatory response profile will not be accompanied by a decline in end-tidal $\mathrm{CO}_{2}$ because the increase in $\dot{\mathrm{VCO}}_{2}$ and $\dot{\mathrm{V}}_{\mathrm{E}}$ will be in proportion. Consequently, the partial pressure of $\mathrm{CO}_{2}$ in arterial blood $(\mathrm{PaCO})$ will remain constant (i.e., isocapnic buffering; iso = same, capnic $=\mathrm{CO}_{2}$ ) (Figure 4). Indeed, during rapid-incremental INC, the decline in $\mathrm{PaCO}$ and endtidal $\mathrm{CO}_{2}$ that characterizes "respiratory compensation" in response to metabolic acidosis will not occur for $\geq 2$ additional min during which work (and metabolic) rate continue to increase (Figure 4) ${ }^{12}$. For this swimmer, the metabolic rates characterizing these distinct changes in gasexchange and ventilatory response driven by the increased contribution of the "anaerobic pathway" to energy demand occurred at $75 \%$ and $86 \%$ of the $\dot{\mathrm{VO}}_{2}$ peak, respectively (Table 1). 


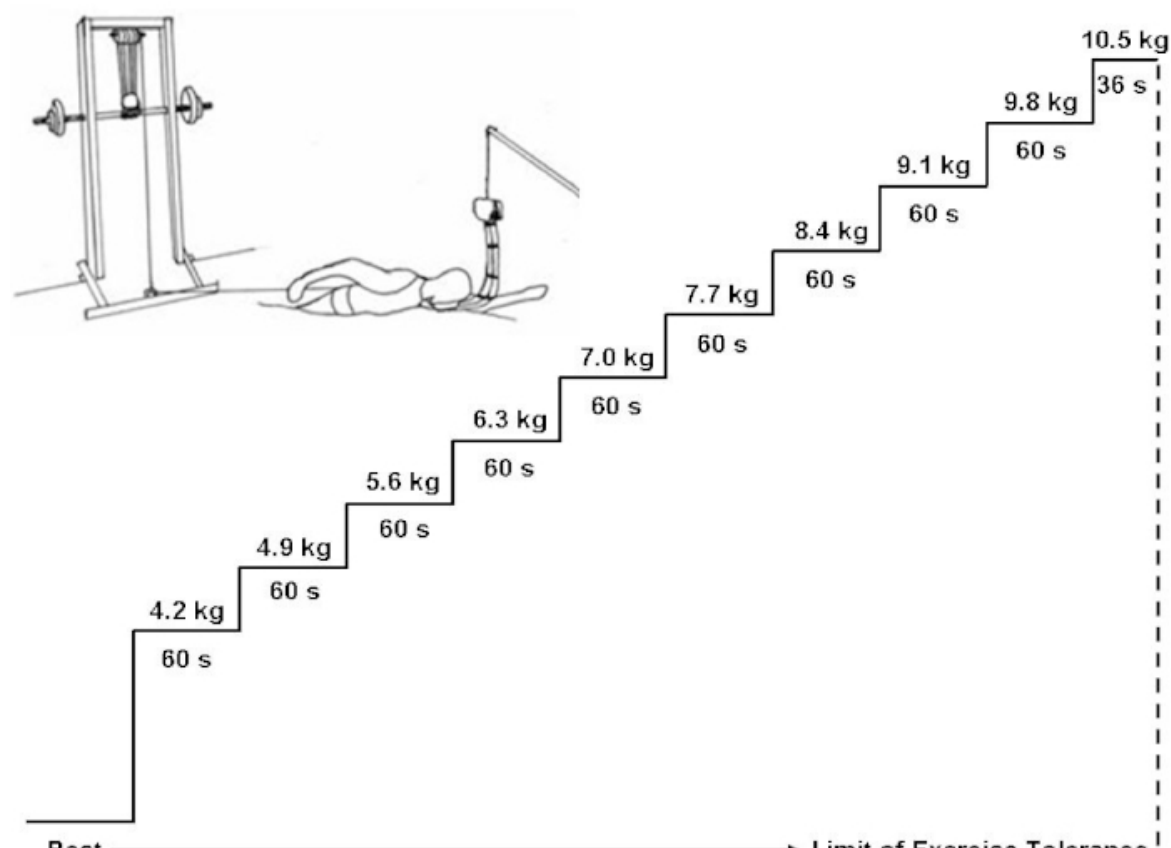

Figure 1: Schematic representation of the rapidly incremented tethered-swimming test that can be used to determine important parameters of cardiorespiratory fitness for swimmers. The loading profile and time to limit of exercise tolerance that are depicted are for a representative subject, a 24-year-old swimmer who competes in short-distance events. Please click here to view a larger version of this figure.

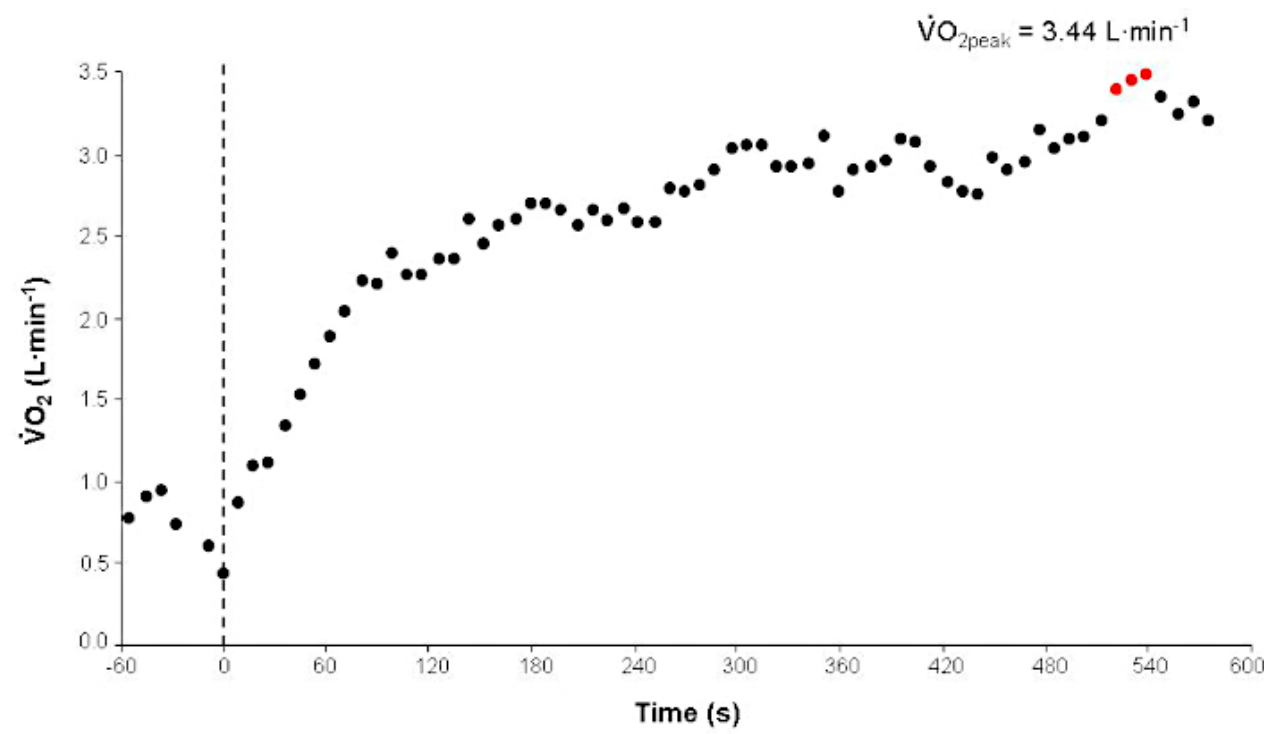

Figure 2: Pulmonary $\mathrm{O}_{2}$ uptake response during the rapidly incremented tethered-swimming test performed by the representative subject. The vertical dashed line represents the point at which load incrementation began. The red circles represent the highest three-point rolling-average rate of $\mathrm{O}_{2}$ uptake that was measured during the test. We defined that value as this swimmer's $\mathrm{VO}_{2 \text { peak }}$ for this form of incremental exercise. Please click here to view a larger version of this figure. 


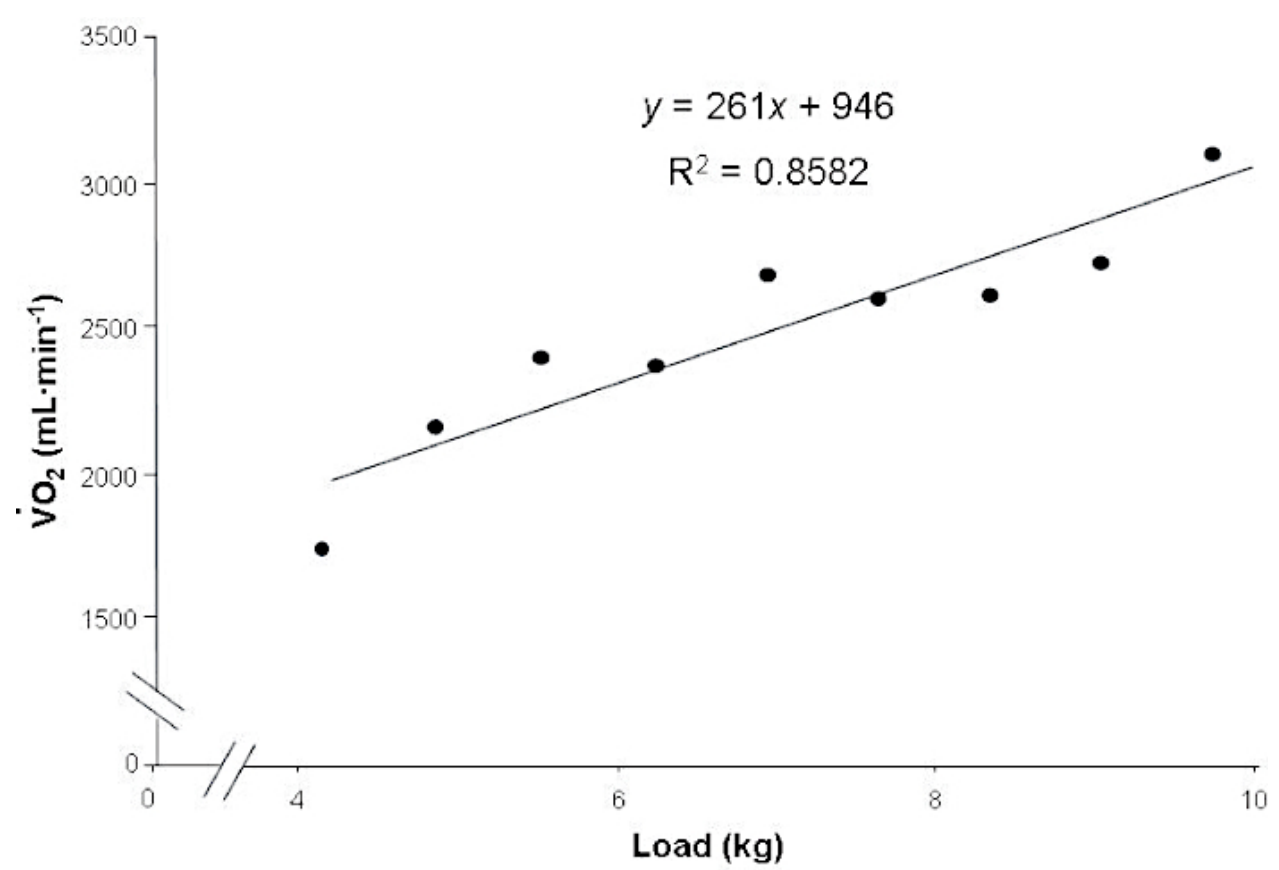

Figure 3: The $\mathrm{VO}_{2}$-load coordinates for the representative subject for each completed stage of the rapidly incremented tethered-

swimming test. Linear regression analysis was used to derive the line of best fit that is depicted. The slope of this line is used as a measure of exercise economy. Please click here to view a larger version of this figure. 


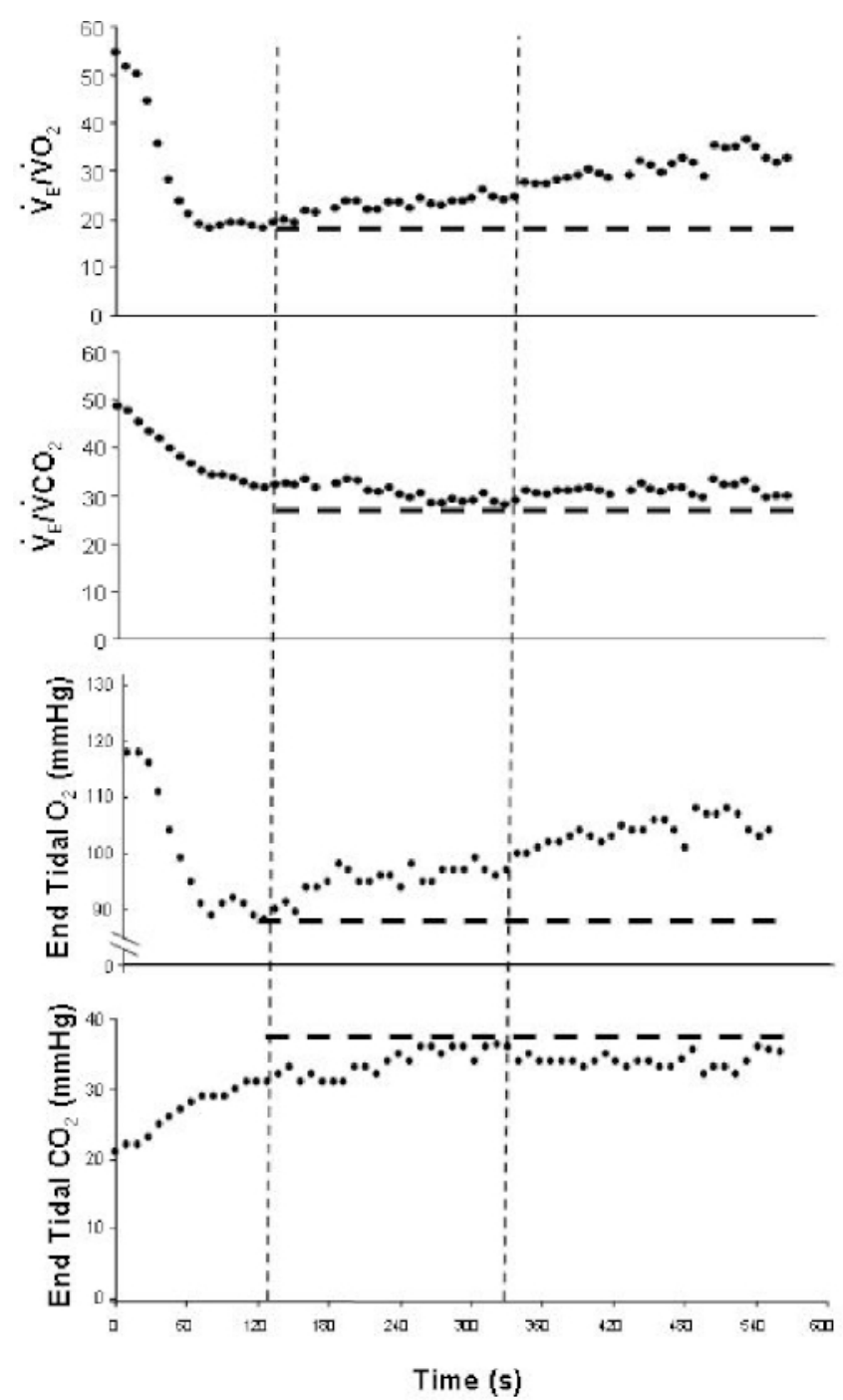

Figure 4: Gas exchange and ventilatory responses for the representative subject during the rapidly incremented tethered-swimming test. From left to right, vertical dashed lines are aligned with the gas exchange threshold and respiratory compensation point, respectively. Horizontal dashed lines are positioned at the nadir (top three graphs) or apex (bottom graph) of data points. See text for details on how these metabolic breakpoints were determined by visual inspection. With slight modification, this figure has been reprinted with permission from the original publishers ${ }^{20}$. Please click here to view a larger version of this figure. 


\begin{tabular}{|c|c|}
\hline Body mass $(\mathrm{kg})$ & 84.7 \\
\hline Stature $(\mathrm{cm})$ & 184 \\
\hline Time to limit of exercise tolerance (s) & 576 \\
\hline Stages completed & 9.6 \\
\hline Peak load (kg) & 10.5 \\
\hline$\dot{\mathrm{V}} \mathrm{O}_{2 \text { peak }}\left(\mathrm{L} \cdot \mathrm{min}^{-1}\right)$ & 3.44 \\
\hline$\dot{\mathrm{V}} \mathrm{O}_{2 \text { peak }}\left(\mathrm{mL} \cdot \mathrm{min}^{-1} \cdot \mathrm{kg}^{-1} \mathrm{BM}\right)$ & 40.6 \\
\hline$\dot{\mathrm{VO}} \mathrm{LGET}_{\mathrm{GE}}\left(\mathrm{L} \cdot \mathrm{min}^{-1}\right)$ & 2.57 \\
\hline$\dot{\mathrm{V}} \mathrm{O}_{2 \mathrm{GET}}\left(\mathrm{mL} \cdot \mathrm{min}^{-1} \cdot \mathrm{kg}^{-1} \mathrm{BM}\right)$ & 30.3 \\
\hline$\dot{\mathrm{VO}}_{2 \mathrm{GET}}\left(\% \dot{\mathrm{VO}}_{2 \text { peak }}\right)$ & 75 \\
\hline$\dot{\mathrm{V}} \mathrm{O}_{2 \mathrm{RCP}}\left(\mathrm{L} \cdot \mathrm{min}^{-1}\right)$ & 2.95 \\
\hline$\dot{\mathrm{V}} \mathrm{O}_{2 \mathrm{RCP}}\left(\mathrm{mL} \cdot \mathrm{min}^{-1} \cdot \mathrm{kg}^{-1} \mathrm{BM}\right)$ & 34.8 \\
\hline$\dot{\mathrm{V}} \mathrm{O}_{2 \mathrm{RCP}}\left(\% \dot{\mathrm{V}} \mathrm{O}_{2 \text { peak }}\right)$ & 86 \\
\hline$\dot{\mathrm{V}} \mathrm{O}_{2}$-load slope $\left(\mathrm{mL} \cdot \mathrm{min}^{-1} \cdot \mathrm{kg}^{-1}\right)$ & 261 \\
\hline$\dot{\mathrm{V}} \mathrm{O}_{2}$-load slope $\left(\mathrm{mL} \cdot \mathrm{min}^{-1} \cdot \mathrm{kg}^{-1} \mathrm{BM} \cdot \mathrm{kg}^{-1}\right)$ & 3.1 \\
\hline
\end{tabular}

Table 1: Cardiorespiratory parameters for the representative subject as measured during the rapidly incremented tethered-swimming test.

\section{Discussion}

An exercise challenge that involves enduring an incremental increase in WR until $T_{\text {lim }}$ is reached is a standard testing protocol for assessment of endurance athletes. When such a test is performed with gradual, but rapid incrementation, it is particularly useful because in addition to the $\dot{\mathrm{V}}_{2 \max }$, gas exchange and ventilatory data collected during the test can be used to distinguish the region bounded by GET and RCP where acidosis is present, but arterial partial pressure of $\mathrm{CO}_{2}\left(\mathrm{PaCO}_{2}\right)$ is maintained ${ }^{14,15}$. The metabolic rates that serve as lower ${ }^{3,6}$ and upper ${ }^{7,8,9,10}$ boundaries of this region approximate those that partition the heavy-intensity domain during CWR.

Generally speaking, the primary parameter of interest derived from the assessment of endurance athletes with $I_{\mathrm{NC}}$ is the $\mathrm{VO}_{2 \mathrm{max}}$, which is used to monitor the athlete's level of cardiorespiratory fitness. Moreover, $\mathrm{V}_{2 \max }$ is often used as a way to assign exercise WR when prescribing training programs (i.e., WR specified as a percentage of $\dot{\mathrm{V}} \mathrm{O}_{2 \max }$ ). However, a growing body of research confirms that the pulmonary gas exchange (and, by extension, muscle metabolic) response to a linear increase in WR is not linear and, importantly, the characteristics of this nonlinearity vary for different individuals (and for the same individual at different levels of conditioning) ${ }^{11}$. Normalizing exercise intensity according to $\mathrm{V}_{2 \max }$ is, therefore, flawed because it does not guarantee a similar level of "metabolic strain" for different individuals ${ }^{11,26,27}$. Conversely, normalizing intensity in relation to the intensity domains that reflect the nonlinearity across the intensity spectrum ensures that a similar metabolic challenge will be encountered. As opposed to $\mathrm{VO}_{2 \max }$, it is, therefore, the metabolic rates that bound the exercise-intensity domains that are important to consider when the objective is to prescribe endurance training in a consistent fashion.

During CWR, metabolic rates situated below $\dot{\mathrm{VO}}_{2 \mathrm{GET}}$ comprise the moderate-intensity domain where a $\dot{\mathrm{V}}_{2}$ steady state can be attained rapidly, muscle metabolic perturbation is minimal and exercise is sustainable for an extended period (e.g., $\sim 4 \mathrm{~h})^{4,5}$. Within this domain, depletion of muscle [glycogen] and impairment in neuromuscular excitability/transmission have recently been implicated as reasons for reaching $\mathrm{T}_{\text {lim }}{ }^{5}$. For metabolic rates above $\dot{\mathrm{VO}}_{2 \mathrm{GET}}$ but below what has been termed the "critical metabolic rate," a steady state $\dot{\mathrm{VO}}_{2}$ is also achievable; however, in this case, attainment is delayed by the presence of a $\mathrm{VO}_{2}$ slow component that increases the $\mathrm{V}_{2}$ cost of work above that which would be predicted by linear extrapolation of the $\mathrm{V}_{2}$ cost of exercise in the moderate-intensity domain ${ }^{28}$. During exercise within this domain, muscle metabolic perturbation (e.g., decreased [phosphocreatine], [ATP], [glycogen] and $\mathrm{pH}$; increased [lactate]) is greater and $\mathrm{T}_{\text {lim }}$ is markedly reduced (e.g., 45 minutes) $)^{5}$. A $\dot{V O}_{2}$ slow component is also present during CWR requiring metabolic rates above the critical metabolic rate (i.e., within the severe-intensity domain); however, in this case, a steady state cannot be achieved as $\dot{\mathrm{VO}}_{2}$ rises inexorably, $\dot{\mathrm{VO}}_{2 \text { peak }}$ intervenes (if exercise is sustained for a long enough period of time), a critical level of substrate depletion and/or metabolite accumulation is reached and $\mathrm{T}_{\text {lim }}$ is imminent in a relatively short period of time (e.g., 2-14 min depending upon work rate) $)^{5}$.

With respect to endurance training for athletes, it is well accepted in both theory and practice that time should be devoted to exercise in each of the intensity domains so that positive adaptations exclusive to work performed in each can be gleaned ${ }^{28}$. For example, a typical week for an endurance athlete might include easy training in the moderate domain, steady training in the heavy domain and tempo and interval training in the

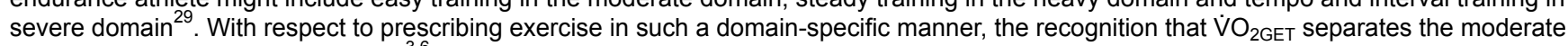
from heavy domain is well accepted ${ }^{3,6}$; hence, moderate-intensity exercise can be prescribed in a normalized manner as a percentage of $\mathrm{VO}_{2 \mathrm{GET}}$ as measured on a rapidly incremented RAMP-INC. Alternatively, controversy exists regarding the critical metabolic rate that establishes the heavy/severe border. Traditionally, the determination of the highest speed or power output that does not cause a rise of blood [lactate] of $>1 \mathrm{mmol}^{-1} \mathrm{~L}^{-1}$ between 10 and 30 min during a series of CWR bouts (i.e., the "maximal lactate steady state;" MLSS) has been used for this purpose $\mathrm{s}^{30,31}$. However, when actual measurements of $\mathrm{T}_{\mathrm{lim}}$ are made by expending the finite capacity for work in the severe domain (W') during a series of CWR bouts or a single all-out bout, it has recently been suggested that the "critical power" (CP) so revealed (i.e., the asymptote 
of the power- $T_{\text {lim }}$ hyperbola for the former testing protocol or the end-test power for the latter) can be greater than the power output indicated by the MLSS assessment ${ }^{32,33,34,35}$. At present, it is safe to conclude that while both CP and MLSS testing provide reasonable estimates of the heavy-/severe-intensity boundary, each of these estimates can be influenced by a number of factors such that congruence between the two is not always present.

In 2017, Keir et al. had subjects perform a multi-bout CP-testing protocol and found that the metabolic rate at CP was statistically similar to the RAMP-INC-derived measurement of $\dot{\mathrm{VO}}_{2 \mathrm{RCP}}{ }^{7}$. The authors concluded that in addition to the metabolic rate at $\mathrm{CP}, \dot{\mathrm{VO}} \mathrm{O}_{2 \mathrm{RCP}}$ might provide an alternative way to determine the critical metabolic rate that separates the heavy from severe domain. However, it is important to recognize that if agreement is present, it is only when the parameters are expressed as metabolic rates because RCP cannot be linked to a specific work rate ${ }^{36}$. Furthermore, given that respiratory compensation can be driven by both exercise WR (i.e., intensity) and the time for which a supra-GET WR is sustained, determining GET and RCP as distinct breakpoints (as opposed to a single "anaerobic threshold" that effectively melds the two) requires INC with relatively rapid incrementation ${ }^{20}$. The clear delineation between the two breakpoints for the representative-subject data we have presented (see Figure 4) verifies that the rapidly incremented tethered-swimming test we are advancing satisfies this criterion.

In addition to discrete values for $\dot{\mathrm{V}} \mathrm{O}_{2 \mathrm{GET}}$ and $\dot{\mathrm{V}} \mathrm{O}_{2 \mathrm{RCP}}$, we have shown that a rapidly incremented tethered-swimming test can be used with sufficient caveats (see above regarding how this value will be specific for a given RAMP-INC protocol and not necessarily indicative of the response that will be present during CWR exercise) to determine the athlete's exercise economy as indicated by the $\dot{V}_{2}$-load slope during the test $^{20}$. This is an important attribute to assess because athletes who are more economical are advantaged during endurance performance. For example, cross-sectional studies indicate that trained athletes possess better exercise economy ${ }^{37}$ while longitudinal studies confirm that exercise economy improves from training ${ }^{38}$. Consequently, deriving this parameter for swimmers from a rapidly incremented tethered-swimming test could be useful both for predicting athletic potential prior to training and monitoring changes that occur as a result of it. However, in addition to recognition of the specificity of this parameter for RAMP-INC (see above), it is important to recognize that only data from the linear-rise portion of the $\dot{\mathrm{VO}}_{2}$ response should be used for this purpose. Conversely, any data reflecting an initial lag in the $\dot{\mathrm{VO}}_{2}$ response (the $\dot{\mathrm{V}} \mathrm{O}_{2}$ "mean response time") and/or a $\mathrm{V}_{2}$ plateau preceding $\mathrm{T}_{\text {lim }}$ should be excluded from the fit.

One important caveat to our contention that the tethered-swimming test we have described can serve as a "swim ergometer" for measuring cardiorespiratory parameters that are relevant for free swimming is that the degree to which the tethered methodology alters technique sufficiently to dissociate the two requires further elucidation. For example, when reporting the highest $\dot{\mathrm{VO}}_{2}$ measured during the test $^{16}$, we are reticent to refer to it as the $\mathrm{VO}_{2 \max }$ because we did not have swimmers also perform a free-swimming INC in our study ${ }^{20}$. Consequently, we cannot confirm that the $\mathrm{V}_{2 \text { peak }}$ during the tethered test is similar to that which is measured using a free-swimming protocol. Although a correlation between the two values has been established ${ }^{39,40,41}$, previous research that compared the two has returned equivocal findings. For example, Bonen et al. reported $\dot{\mathrm{V}} \mathrm{O}_{2 \text { peak }}$ values for free and tethered swimming that were similar and within the range of variation expected for repeat measurement of $\mathrm{VO}_{2 \max }{ }^{40}$ whereas Magel and Faulkner found a lower value for tethered compared to free swimming ${ }^{41}$. The reason(s) for these disparate findings is/are unclear but might reflect the fact that local muscular fatigue and/or ventilatory distress intervened before $\mathrm{VO}_{2 \mathrm{max}}$ was reached for swimmers who were unaccustomed to tethered swimming in the latter study ${ }^{42}$. Regardless of this distinction, future research should be designed to compare $\dot{\mathrm{V}}_{2 \text { peak }}$ values for tethered and free swimming during both INC and supramaximal severe-intensity CWR bouts to $\mathrm{T}_{\text {lim }}$ which are used for confirming that a $\dot{\mathrm{VO}}_{2 \text { peak }}$ measured during INC is indeed the maximum $\dot{\mathrm{VO}}_{2}$ that can be achieved (i.e., "verification bouts") $)^{42}$. Similarly, over the course of the entire test, it is possible that applying load in an incremental manner might result in different "adaptive strategies" by the swimmers in response to the increase of intensity compared to increasing velocity during free swimming. For example, the load might reach a level beyond which biomechanical changes are required that are unlike those that would allow for a more rapid stroke pattern as velocity is increased during free swimming. This could influence the $\dot{\mathrm{VO}}_{2}-\mathrm{WR}$ slope and/or estimation of $\dot{\mathrm{V}} \mathrm{O}_{2 \mathrm{GET}}$ and $\dot{\mathrm{V}} \mathrm{O}_{2 \mathrm{RCP}}$. More research comparing tethered with free swimming is required to provide insight in this regard.

Unlike the speed increases that are used to increment WR during free-swimming INC testing, we have shown that the load increases employed for tethered swimming allow for a gradual, but rapid increase in WR. Consequently, we advance this type of testing as a "swim ergometer" that can be used to determine $\dot{\mathrm{V}}_{2 \mathrm{GET}}, \dot{\mathrm{VO}}_{2 \mathrm{RCP}}$ and exercise economy much like a cycle ergometer is used for performing a smooth ramp protocol ${ }^{14}$. We have also used this test to measure the peak $\mathrm{VO}_{2}$ response; however, how this value compares to the $\mathrm{VO}_{2 \text { max }}$ that is typically assessed during free swimming remains to be resolved.

\section{Disclosures}

The authors have no disclosures to report.

\section{Acknowledgments}

This work was supported by CIPER-Foundation for Science and Technology (FCT), Portugal (UID/DTP/00447/2019) and financed in part by the Coordenação de Aperfeiçoamento de Pessoal de Nível Superior - Brasil (CAPES) - Finance Code 001", and to the São Paulo Research Foundation - FAPESP (PROCESS 2016/04544-3 and 2016/17735-1). Authors would like to thank João Guilherme S. V. de Oliveira to the assistance in data sampling. Mário A. C. Espada acknowledge the financial support from IPDJ - Portuguese Institute of Sports and Youth.

\section{References}

1. Hill, A.V., Lupton, H. Muscular exercise, lactic acid, and the supply and utilization of oxygen. Quarterly Journal of Medicine. 16 (62), $135-171$ (1923).

2. Davis, H.A., Bassett, J., Hughes, P., Gass, G.C. Anaerobic threshold and lactate turnpoint. European Journal of Applied Physiology Occupational Physiology. 50 (3), 383-92 (1983).

3. Beaver, W.L, Wasserman, K., Whipp, B.J. A new method for detecting anaerobic threshold by gas exchange. Journal of Applied Physiology. 60 (6), 2020-2027 (1986). 
4. Whipp, B.J., Wasserman, K. Oxygen uptake kinetics for various intensities of constant-load work. Journal of Applied Physiology. 33 (3) 351-356 (1972).

5. Black, M. I. et al. Muscle metabolic and neuromuscular determinants of fatigue during cycling in different exercise intensity domains. Journal of Applied Physiology. 122 (3), 446-459 (2017).

6. Whipp, B.J. Dynamics of pulmonary gas exchange. Circulation. 76 (6 Pt 2), V118-128 (1987).

7. Keir, D.A. et al. Exercise Intensity Thresholds: Identifying the Boundaries of Sustainable Performance. Medicine and Science in Sports and Exercise. 47 (9), 1932-1940 (2017).

8. Keir, D.A., Paterson, D.H., Kowalchuk, J.M., Murias, J.M. Using ramp-incremental $\mathrm{VO}_{2}$ responses for constant-intensity exercise selection. Applied Physiology, Nutrition and Metabolism. 43 (9), 882-892 (2018).

9. lannetta, D. et al. An equation to predict the maximal lactate steady state from ramp-incremental exercise test data in cycling. Journal of Science and Medicine in Sport. 21 (12), 1274-1280 (2018).

10. lannetta, D., Inglis, E.C., Fullerton, C., Passfield, L., Murias, J.M. Metabolic and performance-related consequences of exercising at and slightly above MLSS. Scandinavian Journal of Medicine \& Science in Sports. 28 (12), 2381-2493 (2018).

11. DiMenna, F.J., Jones, A.M. "Linear" Versus "Nonlinear" $\mathrm{VO}_{2}$ Responses to Exercise: Reshaping Traditional Beliefs. Journal of Exercise Science \& Fitness. 7 (2), 67-84 (2009).

12. Mitchell, J.H., Sproule, B.J., Chapman, C.B. The physiological meaning of the maximal oxygen intake test. Journal of Clinical Investigation. 37 (4), 538-547 (1958).

13. McArdle, W.D., Katch, F.I., Pechar, G.S. Comparison of continuous and discontinuous treadmill and bicycle tests for max VO2. Medicine and Science in Sports and Exercise. 5 (3), 156-160 (1973).

14. Whipp, B.J., Davis, J.A., Torres, F., Wasserman, K. A test to determine parameters of aerobic function during exercise. Journal of Applied Physiology: Respiratory Environmental and Exercise Physiology. 50 (1), 217-221 (1981).

15. Whipp, B.J., Davis, J.A., Wasserman, K. Ventilatory control of the 'isocapnic buffering' region in rapidly-incremental exercise. Respiratory Physiology. 76 (3), 357-367 (1989).

16. Boone, J., Bourgois, J. The oxygen uptake response to incremental ramp exercise: methodogical and physiological issues. Sports Medicine. 42 (6), 511-526 (2012).

17. Sousa, A. et al. Critical evaluation of oxygen-uptake assessment in swimming. International Journal of Sports Physiology and Performance. 9 (2), 190-202 (2014)

18. Fernandes, R.J., Sousa, M., Machado, L., Vilas-Boas, J.P. Step length and individual anaerobic threshold assessment in swimming. International Journal of Sports Medicine. 32 (12), 940-946 (2011).

19. Ribeiro, J. et al. Metabolic and ventilatory thresholds assessment in front crawl swimming. The Journal of Sports Medicine and Physical Fitness. 55 (7-8), 701-707 (2015).

20. Pessôa Filho, D.M. et al. A rapidly incremented tethered-swimming test for defining domain-specific training zones. Journal of Human Kinetics. 57 (1), 117-128 (2017).

21. Dopsaj, M. et al. The relationship between $50 \mathrm{~m}$-freestyle results and characteristics of tethered forces in male sprint swimmers: A new approach to tethered swimming test. Physical Education \& Sport. 1 (7), 15-22 (2000).

22. Cefise biotechnologia esportiva. Produtos. http://www.cefise.com.br/produtos/ (2019).

23. Cefise biotechnologia esportiva. N2000 User Manual. http://www.cefise.com.br/anexos_manuais/90/Manual-N2000.pdf (2019).

24. Wheatley, C.M. et al. Conducting Maximal and Submaximal Endurance Exercise Testing to Measure Physiological and Biological Responses to Acute Exercise in Humans. Journal of Visualized Experiments. 17 (140) (2018).

25. Lansley, K.E, DiMenna, F.J., Bailey, S.J., Jones, A.M. A 'new' method to normalise exercise intensity. International Journal of Sports Medicine. 32 (7), 535-541 (2011).

26. Iannetta, D. et al. A Critical Evaluation of Current Methods for Exercise Prescription in Women and Men. Medicine and Science in Sports and Exercise. [Epub ahead of print] (2019).

27. Scharhag-Rosenberger, F., Meyer, T., Gässler, N., Faude, O., Kindermann, W. Exercise at given percentages of VO2max: heterogeneous metabolic responses between individuals. Journal of Science and Medicine in Sport. 13 (1) 74-79 (2010).

28. Midgley, A.W., McNaughton, L.R., Jones, A.M. Training to enhance the physiological determinants of long-distance running performance: can valid recommendations be given to runners and coaches based on current scientific knowledge? Sports Medicine. 37 (10) $857-880$ (2007).

29. Jones, A.M., DiMenna, F.J. Cardiovascular Assessment and Aerobic Training Prescription. In: Strength and Conditioning: Biological Principles and Practical Applications. M. Cardinale, R. Newton and K. Nosaka, eds. London: John Wiley \& Sons, Ltd. 291-304 (2011).

30. Beneke, R., von Duvillard, S.P. Determination of maximal lactate steady state response in selected sports events. Medicine and Science in Sports and Exercise. 28 (2) 241-246 (1996).

31. Beneke, R.M., Hütler, M., Leithäuser, R.M. Maximal lactate-steady-state independent of performance. Medicine and Science in Sports and Exercise. 32 (6) 1135-1139 (2000).

32. Smith, C.G., Jones, A.M. The relationship between critical velocity, maximal lactate steady-state velocity and lactate turnpoint velocity in runners. European Journal of Applied Physiology. 85 (1-2) 19-26 (2001).

33. Pringle, J.S., Jones, A.M. Maximal lactate steady state, critical power and EMG during cycling. European Journal of Applied Physiology. 88 (3) 214-226 (2002).

34. Mattioni Maturana, F., Keir, D.A., McLay, K.M., Murias, J.M. Can measures of critical power precisely estimate the maximal metabolic steadystate? Applied Physiology Nutrition and Metabolism. 41 (11) 1197-1203 (2013).

35. Jones, A.M, Burnley, M., Black, M.I., Poole, D.C., Vanhatalo, A. The maximal metabolic steady state: redefining the 'gold standard'. Physiological Reports. 7 (10) e14098 (2018).

36. Scheuermann, B.W., Kowalchuk, J.M. Attenuated respiratory compensation during rapidly incremented ramp exercise. Respiratory Physiology. 114 (3) 227-238 (1998).

37. Morgan, D.W., et al. Variation in the aerobic demand of running among trained and untrained subjects. Medicine and Science in Sports and Exercise. 27 (3) 404-409 (1995).

38. Franch, J., Madsen, K., Djurhuus, M.S., Pedersen, P.K. Improved running economy following intensified training correlates with reduced ventilatory demands. Medicine and Science in Sports and Exercise. 30 (8) 1250-1256 (1998).

39. Holmer, I., Lundin, A., Eriksson, B. Maximum oxygen uptake during swimming and running by elite swimmers. Journal of Applied Physiology. 36 (6) 711-714 (1974). 
40. Bonen, A., Wilson, B.A., Yarkony, M., Belcastro, A.N. Maximal oxygen uptake during free, tethered, and flume swimming. Journal of Applied Physiology: Respiratory, Environmental and Exercise Physiology. 48 (2) 232-235 (1980).

41. Magel, J.R., Faulkner, J.A. Maximum oxygen uptakes of college swimmers. Journal of Applied Physiology. 22 (5) $929-933$ (1967).

42. Poole, D.C., Jones, A.M. Measurement of the maximum oxygen uptake VO2max: VO2peak is no longer acceptable. Journal of Applied Physiology. 122 (4) 997-1002 (2017). 